\title{
Ámbitos de investigación de la carrera de administración de empresas: Creación de empresas y gestión de PYMES
}

\section{Fields of research in the career of business administration: Business creation and management of SMEs}

\section{Domínios de investigação na carreira de administração de empresas: Criação e gestão de empresas de PME}

Jorge Oswaldo Quevedo-Vázquez ${ }^{\mathrm{I}}$

joquevedov@ucacue.edu.ec

Lesi Vanessa Giler-Escandón II

lvgilere@ucacue.edu.ec

Xavier Augusto Mantilla-Crespo ${ }^{\text {III }}$

xamantillac@ucacue.edu.ec

Florencio Iván García-Álvarez ${ }^{\text {IV }}$

figarciaa@ucacue.edu.ec

Recibido: 10 de enero de 2019 *Aceptado: 26 de febrero de 2019 * Publicado: 05 de abril de 2019

I. Magíster en Administración de Negocios, Diploma Superior en Práctica Docente Universitaria, Economista, Licenciado en Economía y Finanzas, Docente Universidad Católica de Cuenca, Sede Azogues, Cuenca, Ecuador.

II. Magíster en Administración Tributaria, Diploma Superior en Tributación, Ingeniero en Ciencias Administrativas, Técnico Superior en Administración de Negocios, Docente Universidad Católica de Cuenca, Sede Azogues, Cuenca, Ecuador.

III. Magíster en Administración de Empresas Mención en Recursos Humanos y Marketing, Especialista en Docencia Universitaria, Auditor, Contador Público, Ingeniero Empresarial, Docente Universidad Católica de Cuenca, Sede Azogues, Cuenca, Ecuador.

IV. Magíster en Administración de Empresas Mención en Recursos Humanos y Marketing, Especialista en Docencia Universitaria, Ingeniero Comercial, Docente Universidad Católica de Cuenca, Sede Azogues, Cuenca, Ecuador. 


\title{
Resumen
}

Este artículo presenta un estudio desde el ámbito de investigación de la carrera de Administración de Empresas de la Creación de Empresas y Gestión de las PYMES. La metodología fue de tipo descriptivo, no experimental y documental y el corpus del trabajo lo constituyeron los documentos y revisión del material bibliográficos relacionadas a las teorías de las PYMES. El análisis de la información se hizo a través de la inducción y deducción de los contenidos evaluados, considerando el enfoque hermenéutico como la herramienta de interpretación. Los resultados indicaron que en la actualidad los países sin las PYMES se encontrarían en problemas económicos mayores y los empresarios que toman la decisión de emprender un nuevo negocio es un individuo joven y con un espíritu de líder. Dentro de las conclusiones, se evidencio que un emprendedor para tener éxito debe asumir como prioridad la innovación y la renovación. Por otro lado, se indicó que las PYMES se fortalecen como una posibilidad de mejoramiento de la competitividad.

Palabras clave: PYMES; creación de empresas; gestión y competitividad.

\begin{abstract}
This article presents a study from the field of research of the career of Business Administration of Business Creation and Management of SMEs. The methodology was descriptive, not experimental and documentary and the corpus of the work consisted of documents and review of bibliographic material related to the theories of SMEs. The analysis of the information was done through the induction and deduction of the evaluated contents, considering the hermeneutic approach as the interpretation tool. The results indicated that currently the countries without the SMEs would be in major economic problems and the entrepreneurs who make the decision to start a new business is a young individual and with a spirit of leadership. Within the conclusions, it was evident that an entrepreneur to be successful must take innovation and renovation as a priority. On the other hand, it was indicated that SMEs are strengthened as a possibility to improve competitiveness.
\end{abstract}

Keywords: SMEs; business creation; management and competitiveness. 


\section{Resumo}

Este artigo apresenta um estudo do campo de pesquisa da carreira de Administração de Empresas de Criação e Gestão de Negócios de PMEs. A metodologia foi descritiva, não experimental e documental e o corpus do trabalho constou de documentos e revisão de material bibliográfico relacionado às teorias de PMEs. A análise das informações foi realizada através da indução e dedução dos conteúdos avaliados, considerando a abordagem hermenêutica como ferramenta de interpretação. Os resultados indicaram que atualmente os países sem as PMEs estariam em grandes problemas econômicos e os empresários que tomam a decisão de iniciar um novo negócio são jovens e com espírito de liderança. Dentro das conclusões, ficou evidente que um empreendedor de sucesso deve ter como prioridade a inovação e a renovação. Por outro lado, foi indicado que as PME são reforçadas como uma possibilidade de melhorar a competitividade.

Palavras-chave: PME; criação de empresas; gestão e competitividade

\section{Introducción}

La generación de nuevas PYMES, en opinión de Gómez y Hernández, (2009), expresan que el protagonismo de las PYMES se debe a la importancia que tienen en la economía de los países y por el alto grado de generación de empleo. En consecuencia, las pymes se han vuelto un pilar fundamental en la economía del mundo, de Latinoamérica y en especial en el Ecuador.

La mayoría de los emprendimientos (PYMES) son entre familiares, vecinos, colegas. Para Vásquez (2007), "Impera un elevado nivel de compromiso y motivación de sus integrantes, a la vez que la cercanía al cliente le otorga ventajas de información y mayor flexibilidad para producir pequeñas cantidades, a corto plazo y con determinadas especificaciones" (p.34). Todo lo anterior relata la forma de manejarse internamente las PYMES en dónde se observa que es muy buena debido a que la información no tiene que desencadenarse en tantos niveles. Las pequeñas y medianas empresas de México tienen relación con las del Ecuador, por eso Vázquez (2007), menciona como causa principal del fracaso de las PYMES mexicanas, la ausencia de conocimiento del negocio que se inicia. Se

5 Vol. 5, núm. 2, abril 2019, pp. 03-27

5 Jorge Oswaldo Quevedo Vázquez, Lesi Vanessa Giler Escandón, Xavier Augusto Mantilla Crespo, Florencio Iván García Álvarez 
observa claramente que la falta de conocimiento es el problema de todas las PYMES en general, por lo cual no pueden avanzar o permanecer en el mercado.

Cabe señalar, que los requisitos para conseguir préstamos de estos sitios son muchos y algunos complicados y, en el caso de conseguirse, según el Ministerio de Industrias y Productividad (2012), los obtienen a tasas de interés demasiadas altas. Es evidente entonces que aparte de la falta de conocimiento del negocio existe un problema más que es la dificultad al acceso de financiamiento debido a una serie de requisitos casi imposibles y difíciles de cumplir para nuestros emprendedores ecuatorianos.

En el Ecuador existe a parte del mercado de dinero uno claramente identificado como es el mercado de capitales, que en el papel debería actuar como alternativa válida de financiamiento a largo plazo, sin embargo, este mercado no contempla y normativamente dificulta la participación de las PYMES en dicho escenario. Significa entonces que el mercado de capitales no ofrece ni cumple lo que realmente debería de hacer para nuestros emprendimientos ecuatorianos, así poder generar más empleos, más comercio y aumentar nuestra economía territorial.

En América Latina el impulso a la actividad emprendedora se ha convertido en una de sus principales políticas, teniendo las microempresas y las pequeñas empresas una representatividad del $80 \%$ del total de la economía (Zamora, 2013). En efecto se vuelve a corroborar que de manera general las PYMES se han convertido en el pulmón de la economía latinoamericana.

Los emprendedores ecuatorianos, según Araque (2009), tienen factores que motivan a los empresarios a crear una serie de emprendimientos y estos se dividen en dos grupos: positivos vs negativos y externos vs internos. Entonces aquellos grupos que impide o facilita a los pequeños negocios a seguir adelante con éxito o fracasen por ello deben de estar preparados para satisfacer las necesidades y adaptarse a la demanda del mercado. Ante la situación planteada estos dos grupos anuncian factores tales como el cambio de tecnología, la estabilidad macroeconómica, falta de crédito, falta de personal capacitado, entre otros 
En el modelo de las creaciones o surgimiento de nuevas empresas en opinión de Barba (2006), puede agruparse fundamentalmente bajo tres perspectivas diferentes, en función de la importancia atribuida al empresario emprendedor, al proceso mismo de emprender o a las relaciones diversas que es necesario establecer para emprender. En efecto estas perspectivas diferentes influyen en las decisiones correctas para crear una empresa, con un enfoque organizativo para dar razón por la cual se crea y se gestionan con el interés de aplicar relaciones sociales y culturales, por parte de los empresarios, con el objetivo de obtener apoyo social.

Los estudios realizados que identifican las relaciones entre las características personales del empresario con el resultado de la empresa, en opinión de Gartner. (1989), no dan resultados concluyentes de la empresa o tienen una relevancia escasamente significativa. Por consiguiente, el aprendizaje y la experiencia de cada emprendedor son más relevantes que los atributos personales.

La interacción entre factores personales y sociales, en opinión de Baird, (1988), es como un estado de la mente que dirige la atención de la persona y por tanto la experiencia y la acción hacia un específico objeto (meta) o hacia un camino para lograr algo. Resulta de las intenciones de relacionarse con la sociedad el emprendedor para conocer el objetivo más relevante de la sociedad con el fin de lograr el propósito establecido del empresario.

La innovación social como motor de la creación de empresas, en opinión de O’Connor, (2013), indica que la necesidad de favorecer la creación de empresas y su consideración como motor de crecimiento económico se ha convertido en el objetivo final de una gran parte de organismos públicos. En derivación con estos objetivos se ha puesto énfasis en la creación de empresas para el desarrollo tanto económico como social.

En la importancia de las creaciones de las empresas en opinión de Anokhin et al. (2009), indican que un aspecto esencial para la creación de nuevas empresas es la innovación. En este sentido, los empresarios trabajan de manera paralela, con la innovación, pero también con las iniciativas empresariales de cada emprendedor para obtener ventajas. De esta manera muchos empresarios consideran este hecho importante, es por ello que se ocupan en designar este análisis en sus empresas para el éxito empresarial como social.

7 Vol. 5, núm. 2, abril 2019, pp. 03-27

7 Jorge Oswaldo Quevedo Vázquez, Lesi Vanessa Giler Escandón, Xavier Augusto Mantilla Crespo, Florencio Iván García Álvarez 
La creación de empresas y estrategia tiene una actividad muy importante dentro de la economía de un país y del mundo. El nuevo escenario competitivo presenta nuevas ideas, nuevos problemas y nuevos desafíos (Bettis y Hitt, 1995). Nuevos desafíos en el contexto competitivo implican nuevos retos y esquemas de pensamiento, no sólo en términos empresariales sino también en términos académicos.

Una es la liderada por Porter (1980) desde el paradigma de la organización industrial, y la otra, la correspondiente al enfoque de las capacidades y recursos, con autores como Wernerfelt (1984), Barney (1991) y Peteraf (1993). La creación de empresas es un área de investigación científica, pero que, al igual que muchas otras disciplinas administrativas, puede ser abordada desde diferentes vertientes teóricas $\mathrm{El}$ enfoque de las capacidades y recursos reconoce que la creación de empresas hace parte de sus fundamentos teóricos, pero sin que ello haya sido objeto directo de estudio. Tal perspectiva se ha concentrado en el análisis de la relación capacidades centrales - entorno, desde el punto de vista de la ventaja competitiva, y toca sólo tangencialmente lo relacionado con la creación de empresas.

Un análisis de los Factores Sociodemográficos determinantes en la creación de empresas en el marco de la interculturalidad en el sector europeo, pero para despertar este interés, primero fue necesario que investigadores y gobiernos reconocieran que el Estado solo no es capaz de generar los niveles adecuados de producción y empleo, sino que es conveniente que la mayor parte de los mismos proceda de la iniciativa privada (Loutfi, 1991; Gelderen, Bosma y Thurik, 2001). Los posibles motivos para generar este interés son; la ralentización del crecimiento económico y la paralela agudización del problema del desempleo; la constatación de la importancia que, para el crecimiento económico y el desarrollo de un país, poseen las capacidades empresariales de sus individuos

Y es que el estado de las causas y las consecuencias de los factores que inciden en la variación de la tasa de creación de empresas, es una cuestión de interés científico y de gran importancia para la política económica y social (Gelderen, et ál., 2001; Díaz, 2005). Los factores que determinan la creación y consolidación empresarial, aquéllos relacionados con el empresario ya que en el surgimiento de nuevas empresas el individuo es el centro sobre el cual gira este proceso, sin él sería imposible desarrollar la puesta en marcha de una empresa. Pérez Urrego (2003), explica que se puede

8 Vol. 5, núm. 2, abril 2019, pp. 03-27

Jorge Oswaldo Quevedo Vázquez, Lesi Vanessa Giler Escandón, Xavier Augusto Mantilla Crespo, Florencio Iván García Álvarez 
establecer que las PYMES o también conocidas como pequeñas y medianas empresas tienen un porcentaje muy alto de empresas a nivel de todo el mundo, y se demuestra que dentro de un país cubren la gran parte de las actividades económicas, al momento de crear algún negocio siempre se empieza estructurando de una forma pequeña para así ir desarrollándose con el paso de los años y puedan llegar a formar una gran empresa.

Valencia, (2007), indica que en las empresas existen problemas originados por los factores externos como la economía, política y tecnología, también se pudo identificar deficiencias internas por ejemplo de mercadeo, producción y personal. El mayor reto de una empresa particularmente las PYMES que laboran en un entorno activo es la expansión de sus competencias internas la cual las llevara al éxito de dicha empresa. Uno de los retos de las empresas, particularmente de las PYMES que operan en entornos dinámicos, es la reproducción de sus competencias internas y del aprendizaje colectivo, lo cual les permitirá ser exitosas y sobrevivir o fracasar (Valencia, 2007). Esa reproducción deriva de la capacidad de la empresa para generar los diferentes recursos necesarios para cumplir con las metas que se hayan trazado.

Birley, (1987), señala que crear nuevas empresas es un elemento clave para el incremento económico, dicho de otra manera, requieren de ciertas condiciones como por ejemplo políticas, sociales, económicas, sensibilidad, agudeza y tino, ya que son organizaciones con un reducido número de colaboradores - empleados, el impacto de una decisión o acción bien o mal ejecutada se expandirá en muy poco tiempo y pondrá en riesgo la operación. Los reportes sobre las Pequeñas y Medianas Empresas (PYMES) según (SBA, 2001), se pueden clasificar en dos tipos, los reportes sobre estadísticas y los reportes sobre Política Industrial. En ambos reportes se reconoce que las PYMES son importantes para la economía y para el desarrollo del país, por el número de empleos que representan y por su contribución a la derrama económica en el mercado.

Además, para los autores Barceló y Pérez, (2003), las áreas de oportunidad en las PYMES son, por ejemplo: el desarrollo de una estrategia de negocio, realizar benchmarking, estudios de mercado, capacitación para la exportación, apoyos de financiamiento para el crecimiento, cursos de administración y de las operaciones del negocio, capacitación del recurso humano en todas las áreas

9 Vol. 5, núm. 2, abril 2019, pp. 03-27

9 Jorge Oswaldo Quevedo Vázquez, Lesi Vanessa Giler Escandón, Xavier Augusto Mantilla Crespo, Florencio Iván García Álvarez 
y desarrollo de sistemas informáticos internos. En consecuencia, las PYMES presentan un factor importante en la actividad económica, hay que tener en cuenta que la gestión de PYMES es un recurso imprescindible al momento de iniciar un negocio, la misma se caracteriza por tener un contenido amplio de medios estratégicos que generan la competitividad. Cuando las pequeñas y medianas empresas presentan problemas es recomendable analizar el mercado para poder innovar y por ende se obtendrá ganancias o ingresos.

En opinión de Solleiro y Castañón (2005), la competitividad es un concepto complejo sobre el cual no existe una definición única, en parte debido a que su utilidad reside en la posibilidad de identificar vías para fomentar empresas nacionales que contribuyan a la elevación real de los niveles de bienestar. Es evidente que para muchas personas la creación de empresas puede generar problemas, una de ellas y la más reconocida es la competitividad, no presenta un concepto concreto, pero se puede referir a la creatividad y capacidad que tienen para producir bienes y servicios de modo eficaz, la ventaja está en ofrecer un producto de buena calidad a un precio razonable. En atención a los planteamientos propuestos, en este artículo se presenta un estudio desde el ámbito de investigación de la carrera de Administración de Empresas, la Creación de Empresas y Gestión de las PYMES.

\section{Desarrollo}

La microempresa y la pequeña empresa tienen una representatividad en la economía cuencana del $80 \%$, porcentaje que coincide con la economía ecuatoriana (Banco Central del Ecuador, 2011) y economías de países latinoamericanos (Zamora, 2013). Sobre las bases de las consideraciones anteriores se nota que aquellos emprendimientos poco a poco han ido creciendo y se convirtieron en un pilar solido en la economía de cualquier país.

\section{Aspectos personales del emprendedor}

A nivel nacional el $83 \%$ de los emprendedores son de sexo masculino (Araque, 2009), cifras parecidas en la ciudad de Cuenca que alcanzan un 78,5\% Cordero et al. (2011). Según se ha visto en los estudios realizados a través de encuestas, entrevistas y recolección de datos, que a pesar de todos los logros y esfuerzos de la mujer por ser reconocida en la sociedad aún sigue predominando el hombre en el

10 Vol. 5, núm. 2, abril 2019, pp. 03-27

Jorge Oswaldo Quevedo Vázquez, Lesi Vanessa Giler Escandón, Xavier Augusto Mantilla Crespo, Florencio Iván García Álvarez 
control de los negocios, ellos son en un porcentaje mayoritario que tienen la iniciativa y asumen el riesgo de emprender.

De acuerdo con Araque (2009), el emprendedor ecuatoriano inicia su empresa mayoritariamente entre 26 y 32 años. Es evidente entonces que a esa edad cuenten con un título universitario, y algunos tienen experiencia por haber trabajado en áreas relacionada a sus ramas de estudio en especial los emprendedores cuencanos deciden abrirse de sus trabajos y se ven motivados a emprender su propio negocio. Araque (2009), señala la misma motivación como la influencia de mayor grado en el emprendedor ecuatoriano. Con referencia a lo anterior, de la oportunidad de emprender un negocio, se dice que existen factores que influyen en las actividades empresariales de los cuencanos y empresarios en general como ejemplo la persistencia (acciones constantes y repetitivas), eficienciacalidad y cumplimiento. McClelland (1961) en su estudio concluye principalmente que: "fijar metas" deber ser la pauta con mayor puntuación por encima de "persistencia".

\section{Condiciones del entorno}

En América Latina, el financiamiento está disponible sólo para firmas maduras menciona Kantis (2004). Se observa claramente que para emprender unos de los mayores obstáculos es la falta de financiamiento por las instituciones bancarias, por eso el $81 \%$ de los cuencanos se financian con sus propios recursos económicos, todo esto de la falta de financiamiento ocurre de igual forma en América Latina.

Araque (2009) menciona que, de acuerdo a una breve investigación, el 60\% de los emprendedores en la ciudad de Quito tienden a financiar sus proyectos con recursos propios y que no existen líneas de crédito orientadas a financiar proyectos nuevos. En este mismo orden y dirección, cabe recalcar que las experiencias de trabajos anteriores, la información del negocio a emprender y la confianza basada en capacitaciones que posee el futuro emprendedor le permite correr menos riegos al momento de decir montar su propia empresa. Arteaga y Lasio (2009), manifiesta que en el año 2009 el 85\% de los emprendedores creía poseer el conocimiento, habilidades y la experiencia para iniciar un nuevo negocio.

11 Vol. 5, núm. 2, abril 2019, pp. 03-27 
El $60.13 \%$ de empresas ecuatorianas manifestaron haber encontrado dificultades con la documentación que exige la banca como requisito.( García et al, 2017). Se observa claramente que estas dificultades las empresas lo manifestaron en grado de mayor a menor importancia tales son: el excesivo número de documentos, los elevados costos de elaboración de los documentos y por último el tiempo que toma la elaboración del mismo.

Las fuentes de financiación preferida por las PYMES es la bancaria en $74.84 \%$, seguida por las cooperativas en $17.68 \%$, pero esas empresas (la banca) no la consideran por diferente razones (García et al., 2017). En ese mismo sentido se menciona que esas razones comprenden a la viabilidad comercial, las garantías y credibilidad de estados financieros, el sector al que pertenecen, el riesgo global asumido por la PYMES y la experiencia en operaciones anteriores.

Tabla 1. Preferencias de financiación

\begin{tabular}{lrrr}
\multicolumn{1}{c}{ Institución } & Frecuencia & absoluta & Frecuencia \\
Bancos & 229 & & relativa \\
Bolsa de valores & 1 & $74,84 \%$ \\
Cooperativas & 54 & $0.33 \%$ \\
Mutualistas & 10 & $17.65 \%$ \\
Propia empresa y otros & 13 & $3,27 \%$ \\
\end{tabular}

Fuente: García et al. (2017)

\section{Sistema de desarrollo emprendedor}

El estudio Global Entrepreneurship Monitor Ecuador (GEM Ecuador) (Lasio, Caicedo y Arteaga, 2009) reveló que más del 75\% de los ecuatorianos invierten en los negocios de familiares, amigos o vecinos. Significa entonces que la mayoría de emprendimientos son financiados por recursos propios debido a todas las trabas que la banca presenta para financiar a las Pymes. Kantis et al. (2004) concluyeron que las redes juegan un papel distintivo en el caso de los emprendedores de empresas dinámicas, para acceder a recursos no monetarios como información, tecnología, materia prima o instalaciones, entre otros.

12 Vol. 5, núm. 2, abril 2019, pp. 03-27 


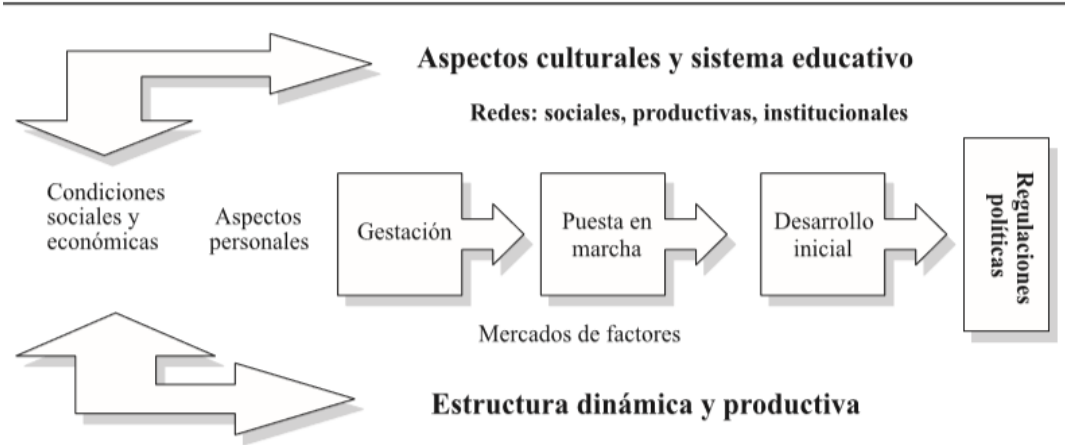

Figura 1. Sistema de desarrollo emprendedor.

Fuente: Kantis et al. (2004).

Barba (2006), plantean las siguientes características del emprendedor: deseo de independencia, mayor predisposición al riesgo, alta necesidad de logro y mayor preferencia por la innovación. Sobre las bases de las consideraciones anteriores se observa que es necesario tener actitud y aptitud para querer ser un empresario de éxito.

Como señalan York and Venkataraman, (2010), en “el momento actual los empresarios contribuyen, en muchos casos, a ayudar a las instituciones existentes en el logro de sus metas, crean nuevos productos sostenibles, y complementan los servicios que las instituciones no pueden desarrollar"'(p.452). En efecto por esos motivos las pymes se han vuelto muy importantes en todos los países. La proactividad consiste en anticiparse a los problemas futuros, necesidades o cambios. La iniciativa remite al acercamiento activo al trabajo (Frese y otros, 1996). Tal como se observan estos puntos son primordiales para que los emprendimientos sigan manteniéndose a través del tiempo y adquieran un buen prestigio en el mercado donde se desarrolla.

Marshall (1963), por su parte, afirmó que podemos dividir a los patrones y demás empresarios en dos clases: los que inauguran nuevos métodos modernos de negocio y los que siguen por caminos trillados. Ante la situación planteada se tiene que fomentar más la innovación y creatividad para lograr tener un éxito asegurado y no seguir los mismos esquemas de la mayoría de emprendedores. En esta línea, para Kirzner (1999) los emprendedores generan cambios dentro de una situación ya existente, porque se orientan por las ineficiencias que otros no han percibido y sobre las cuales existe una ganancia

13 Vol. 5, núm. 2, abril 2019, pp. 03-27

Jorge Oswaldo Quevedo Vázquez, Lesi Vanessa Giler Escandón, Xavier Augusto Mantilla Crespo, Florencio Iván García Álvarez 
potencial. A lo largo de los planteamientos hechos se nota la variedad de pensamientos que indican las inclinaciones o preferencias de los emprendedores al momento de crear su empresa.

Dicen Chandler y Takashi (1994), en virtud de dos funciones básicas que cumple el emprendedor innovador: analizar el entorno, seleccionando oportunidades de negocio y formulando estrategias para aprovecharlas, e interactuar con el entorno para adquirir y utilizar los recursos necesarios para la implementación de la oportunidad. En ese mismo sentido se analiza que antes de emprender es necesario tener un plan de estudio del mercado para así tener más confianza en lo que se va a poner en marcha tratando de arriesgar lo menos posible.

Como plantean Greening y Johnson (1996), existe la imperante necesidad de una estructura conceptual para investigar la importancia de la personalidad y los valores personales de los creadores de empresa, conociendo sus implicaciones en la estrategia y en la toma de decisiones. Todo lo anterior hace referencia que según las estrategias y decisiones que tomen estos futuros empresarios pueden llegar a ser los agentes de cambio en las próximas décadas. Kent (1990), señala que el emprendedor puede ser por factores genéticos, o del entorno. Es evidente entonces que cada ser humano tiene su esencia y pensamientos que se van formando con la educación y el pasar de los años logrando cristalizar sus ideas en sus propios negocios.

Si bien la creación de empresas surge como campo de estudio en la década de los setenta, con su aporte sobre riesgo e incertidumbre, con su idea de nuevas combinaciones y olas de destrucción creativa, con la idea de servicios emprendedores y oportunidades productivas. En esta misma perspectiva sobresalen los economistas austríacos al hacer evidente la importancia del individuo en la economía. Además, introduce el aprendizaje mutuo y la conciencia de participación en el mercado. En la versión de Kirzner (1999), los individuos actúan en función de la búsqueda de oportunidades, es decir, en vez de actuar como maximizadores permanecen alertas a los cambios o irregularidades presentadas en la economía. Se dice que la visión austríaca de la acción humana creativa y activa es contraria a la visión neoclásica, que ve al hombre como un ser pasivo, racional y mecánico dentro de un contexto de mercados eficientes. 
La creación de empresas nace en 1974, este hecho se ve fortalecido por los hallazgos de David Birch (1979), los cuales muestran la creación de pequeñas empresas como el motor del crecimiento económico en Estados Unidos. Los resultados de Brich fueron importantes porque hasta ese momento se asumía que las grandes empresas eran las que generaban el empleo productivo. En base de las condiciones anteriores la creación de empresas podría estar en el abordaje de preguntas relacionadas con el nacimiento, crecimiento, fracaso y transformación de organizaciones, lo mismo que en el contexto y características bajo las cuales surgen los emprendedores. En este sentido, debe ser entendido como un proceso dinámico, de naturaleza cognitiva y en constante interacción con el entorno (Veciana, 1999).

El vínculo entre creación de empresas y estrategia se hace evidente en la década de los noventa con la incorporación de la "rapidez" y la "acción" como elementos estratégicos, lo que llevó a la definición de economía emprendedora. La administración estratégica pasa así de modelos industriales estáticos y mercados eficientes a modelos más dinámicos de cambio y flexibilidad. Las diferencias de desempeño no sólo se atribuyen, entonces, a factores ambientales o industriales sino a competencias distintivas o recursos específicos poseídos por los individuos. Se habla de acciones emprendedoras, acciones estratégicas o, más aun, de emprendimiento estratégico tratando de caracterizar la forma como las empresas deben competir en ambientes dinámicos y con gran incertidumbre.

La administración es un proceso que trata con el trabajo emprendedor de la organización, con la renovación y el crecimiento, particularmente con el desarrollo y utilización de la estrategia que es la guía para las operaciones de la organización. El empresario al igual que el resto de las personas, aprende de sus éxitos y fracasos, por tanto, la combinación de experiencias positivas y negativas moldea el conocimiento del fundador del negocio, incidiendo en sus modelos cognitivos y en la forma como él procesa la información.

El fundador con experiencia tanto en creación de empresas como directiva o en el sector de actividad está asociado positivamente al nacimiento de empresas y en la consolidación empresarial. Desde este enfoque se considera que los empresarios que poseen este tipo de experiencia reconocen un mayor número de oportunidades de negocio más innovadoras que sus homólogos sin experiencia

15 Vol. 5, núm. 2, abril 2019, pp. 03-27

15 Jorge Oswaldo Quevedo Vázquez, Lesi Vanessa Giler Escandón, Xavier Augusto Mantilla Crespo, Florencio Iván García Álvarez 
empresarial. Y es que los empresarios con experiencia directiva o laboral anterior a la creación de la empresa, prestan una mayor atención a la identificación de oportunidades a través de la lectura sobre el sector de actividad. Así mismo, estos empresarios son más propensos a emprender actividades relacionadas con las desempeñadas anteriormente en especial, con aquéllas denominadas "técnicas" (Quintana, 2001). El grado de experiencia de un empresario en creación de empresas y/o en actividades técnicas o directivas influye positivamente en la puesta en marcha de la empresa.

Tanto la edad como la educación han sido frecuentemente asociadas con la creación de nuevas empresas. Algunos investigadores indican que la edad del empresario puede servir para determinar dos fuerzas relacionadas con la actuación empresarial; la experiencia y la vitalidad. Es decir, por un lado, existe la creencia de que los individuos de mayor edad tienen la ventaja de la acumulación de experiencia. Pero también se considera que los individuos más jóvenes poseen la energía, el vigor y el entusiasmo propios de su juventud, que, unidos a la posesión de conocimientos recientes y a esquemas mentales más abiertos, les permiten buscar, reconocer y desarrollar oportunidades de negocio más innovadoras y, por tanto, con mayor potencial de crecimiento económico.

Como fue señalado por Kachlami y Yazdanfar (2016), cerca del 95\% de todas las empresas del mundo son PYMES. Según se ha visto que han ido aumentando el número de emprendimientos ecuatoriano y también a nivel latinoamericano. Kachlami y Yazdanfar, (2016), explican que el crecimiento de la empresa se puede explicar utilizando cuatro perspectivas teóricas diferentes: la perspectiva de la adaptación estratégica, la perspectiva de la motivación, la perspectiva de la configuración y la perspectiva basada en los recursos. En relación con este último es importante recalcar que siempre existirán otros factores que influyan en el crecimiento de las pymes como ejemplo las políticas de estado, el marco legal, la economía, entre otros.

Las políticas hacia las PYMES han ido ganando mayor importancia en América Latina con sistemas de apoyo más estructurados en los distintos países (Belmar y Maggi, 2010; Brown, 2010; Sztulwark, 2010 y Zuleta, 2011). Significa entonces que estas políticas de los diferentes gobiernos deben de apoyar primordialmente a los emprendimientos, pero hasta ahora no están cumpliendo con su función. 
Dom. Cien., ISSN: 2477-8818

Vol. 5, núm. 2, abril 2019, pp. 03-27

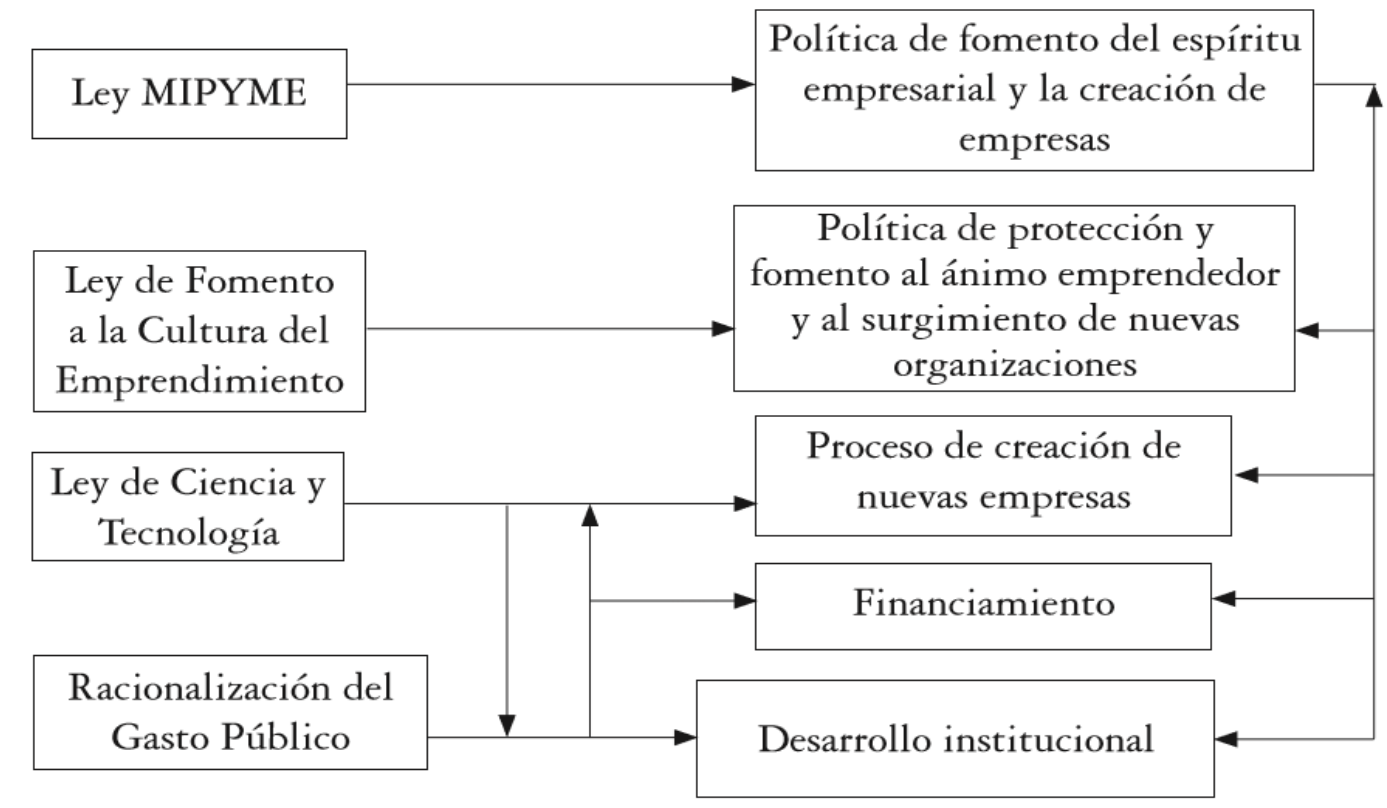

Figura 2. Medidas de apoyo a la creación de empresas.

Fuente: Gómez et al. (2009).

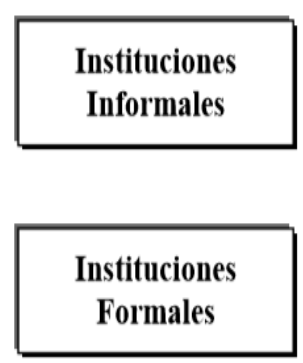

Figura

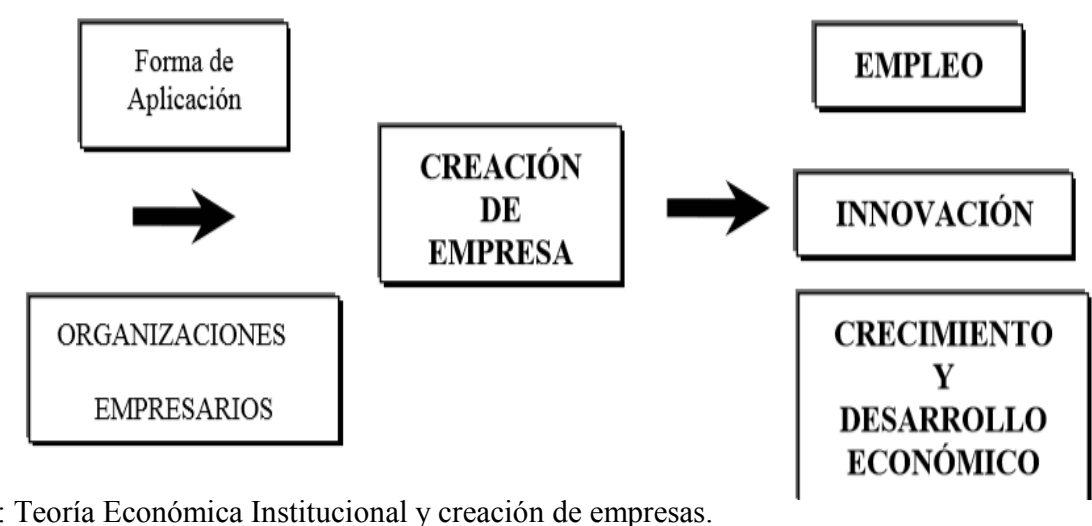

Fuente: Díaz et al. (2005).

Conner (1991), Rumelt (1987) y Wernerfelt (1984) definen la empresa como una colección única de recursos y capacidades, que no se pueden comprar y vender libremente en el mercado, y se enmarca dentro del denominado análisis estratégico interno de la organización (determina fortalezas y debilidades) (Navas \& Guerras, 1998). Con referencia a lo anterior se puede tener una idea más clara de que es una empresa y que debe de tener planes, proyectos y estrategias futuras.

17 Vol. 5, núm. 2, abril 2019, pp. 03-27 
Polanyi (1962, citado en Zapata, 2004) señala que el conocimiento tácito es la clave para la empresa y se centra en un nivel individual bajo la forma de habilidad técnica; esto es, el know how (saber cómo) de los individuos. Es evidente entonces que aparte de todo lo técnico que debe de tener una empresa para poder constituirse es fundamental también tener ese espíritu de líder y administrador.

En dictamen de (Garrido, 2001), los estudios económicos identifican ciertos polos con más desarrollo económico que en el resto del país, por ejemplo: el Distrito Federal, Guadalajara y Monterrey, por lo que en principio una solución general para las PYMES no debería aplicarse en todas las regiones por igual, ni sin tomar en cuenta el sector al que pertenecen, comercial, manufactura o de servicios, ya que esto plantea problemas de gestión propios al producto o servicio, mercados y clientes. De acuerdo con lo antes dicho el autor anuncia que para implementar una empresa o negocio es importante realizar un estudio de mercado para conocer las necesidades de quieres lo operan y luego emprender ya que es un recurso que dependerá el avance o fracaso de la empresa.

El artículo de Kauffman, (2005), analiza la problemática que enfrentan las PYMES mexicanas ante la Globalización y menciona que a nivel interno las PYMES carecen de un sistema de planeación, organización, administración, y controles eficientes, así como de tecnologías propias para la gestión y desarrollo de sus actividades productivas. En dictamen de González, (2015), los estudios para identificar los problemas de las PYMES mezclan dos tipos de enfoques que llamaremos enfoques "externo" e "interno"; el enfoque externo se caracteriza por problemas que afectan a las PYMES, pero que son más bien del tipo macroeconómico y en donde las PYMES no tienen influencia en su solución (o no es su razón de ser); mientras que el enfoque interno, en cambio, se caracteriza por problemas derivados de la gestión propia del negocio y del sector económico al que pertenecen, y donde la PYMES es responsable de su solución y mejora.

En el mundo empresarial se establece una contraparte, es decir positivas o negativas, es por ello que es importante e infaltable antes de crear una empresa considerar las causas y consecuencias que se presentaran en el futuro, para que la empresa tenga vigencia durante años es recomendable aplicar innovación y renovación, se requiere con ello significar una competitividad y asegurar el progreso de la empresa .de esta manera, tomando en consideración lo antes expuesto se identifican las siguientes

18 Vol. 5, núm. 2, abril 2019, pp. 03-27 
factores: a) Contabilidad. Escasez de registros contables, costos mal determinados y precios que no cubren los costos totales. b) Finanzas. Falta de conocimiento para analizar los estados financieros, falta de acceso a créditos convenientes para la empresa y c) Fiscal. Excesiva regulación fiscal, desconocimiento de sus obligaciones y cumplimiento.

El artículo de Sánchez, (2003) menciona un estudio de Nacional Financiera que reporta los motivos o causas internas por las cuales mueren las PYMES, entre las cuales se mencionan: problemas administrativos (43\%), problemas fiscales (16\%) incapacidad de cumplir requisitos (15\%), deficiencia en comercialización (11\%), por errores en insumos y producción $(8 \%)$ y debido a falta de financiamiento (7\%). Existe una variedad de pequeñas y medianas empresas en el mundo, las cuales son creadas con el objetivo de satisfacer las necesidades del cliente o consumidor, por ende recibirá un beneficio económico, sin embargo para que exista una mayor producción de productos se debe llevar un organigrama de la empresa o negocio ya que el personal estará informado de la función que debe realizar, además de que el producto sea de buena calidad y a un precio razonable garantizar una mayor producción o ventas, también las empresas tienen responsabilidades con la población tanto específicas como generales, por ejemplo pueden generar fuentes de trabajo, productividad y con ello el progreso del pueblo, asimismo es importante procurar ganancias para cubrir gastos y alcanzar una buen ascendencia dentro de la sociedad. Cabe recalcar que una entidad se clasifica de acuerdo al sector que pertenece (privado, público, mixto), su organización de capital y su constitución legal. El $50 \%$ de las empresas exportadoras en el país se corresponden a pequeñas y medianas empresas (PYMES), pero apenas 18,6\% de ellas vende con éxito en el exterior en participación de Comercio.

Las PYMES latinoamericanas, enfrentan diversos obstáculos, Melo (2003) presenta tres de los problemas, estrechamente relacionados entre sí, que requieren la máxima atención de la política pública si se quiere que el país mejore su competitividad: i) la inestabilidad en las reglas de juego para la actividad económica privada, ii) la corrupción en la administración pública, y iii) las limitaciones del sistema judicial. También la escasez y el costo del crédito, el escaso desarrollo del mercado de capitales y, posteriormente, la necesidad de mayor innovación.

19 Vol. 5, núm. 2, abril 2019, pp. 03-27 
De allí que la creación de empresas, requiere de varios recursos que fortalezcan a la misma y muestren una proyección futura, la competitividad es un factor positivo razón por la cual ayuda a evitar que las empresas tengan un mismo mecanismo de trabajo o atención al cliente , por lo tanto la, creatividad, diseño, son armas que benefician al desarrollo del negocio ya que si no existe innovación no atraerá la atención de la gente lo que provoca un cierre de la empresa además generando perdida de lo invertido.

Bejarano J. (1995), realizó un estudio acerca de la relación entre cadenas productivas y competitividad, en el cual se hace una descripción del concepto de cadena productiva y aquellos términos relacionados con ella. Este autor vio en las cadenas productivas la mejor forma de aprehender los elementos del sistema pertinentes para el análisis de la competitividad. Así mismo, relacionó el concepto de cadena productiva con un campo de la organización económica, en el cual se genera una serie de eslabonamientos entre conjuntos de unidades de producción, los cuales participan tanto en la producción como en la distribución del producto desde que aparece como materia prima, hasta que sale del campo o es consumido. En esta medida, se afirma que la combinación de relaciones verticales y horizontales, las alianzas y rivalidades profesionales, la competencia por segmentos definidos de mercado y por ganancias, es lo que forma una cadena productiva.

La cadena productiva es un mecanismo que podemos realizar operaciones de insumos en bienes o servicios mediante un proceso tecnológico, es decir se encarga del diseño, entonces se debe aplicar esa técnica ya que facilitara enfrentar problemas como la competitividad, como bien conocemos la empresa es una unidad de producción económica, es diferenciada por la marca o logotipo de la región , además en la actualidad se trabaja con medios tecnológicos que realizan publicidad para generar más ganancias es decir una empresa puede establecer una red de intervención PYMES con el propósito de sacar ventaja a los nuevos productos o servicios innovadores que éstos estén desarrollando. A través de las licencias, un empresario puede tener acceso a personas especializados de marketing y finanzas de las grandes compañías e inclusive conseguir capital que apruebe asociarse a nuevos compradores.

\section{Metodología}

20 Vol. 5, núm. 2, abril 2019, pp. 03-27

Jorge Oswaldo Quevedo Vázquez, Lesi Vanessa Giler Escandón, Xavier Augusto Mantilla Crespo, Florencio Iván García Álvarez 
Se realizó un estudio de tipo descriptivo, no experimental y documental en relación a la Creación de Empresas y Gestión de PYMES. El corpus del trabajo lo constituyeron los documentos y revisión del material bibliográficos relacionadas a los PYMES. El análisis de la información se hizo a través de la inducción y deducción de los contenidos evaluados, considerando el enfoque hermenéutico como la herramienta de interpretación.

\section{Conclusiones}

Posterior al análisis de las teorías y planteamientos propuestos, fue posible expresar el siguiente análisis de los contenidos, a través de las siguientes conclusiones:

- Todas las investigaciones confirman que en la actualidad los países sin las PYMES se encontrarían en problemas económicos mayores, sin poder producir y generar menores costos estatales y nuevas tasas de empleos para que se pueda mejorar la calidad de vida para los ciudadanos en general. Como consecuencia deben de implementar programas de financiamiento, colaboración y apoyo para los emprendimientos y ejecutar los controles respectivos para que cumplan con todo su plan de trabajo.

- Los resultados muestran que el empresario que toma la decisión de emprender un nuevo negocio es un individuo joven, menor de 44 años, con estudios secundarios o superiores y con experiencia en el sector de actividad. Una empresa operando en forma organizada, combina con la técnica y los recursos para elaborar productos o prestar servicios con el objeto de colocarlos en el mercado para obtener una ganancia. De esta manera las pequeñas y medianas empresas deberían de hacer un tipo de asociación y poco a poco hacerse más grande para poder constar y utilizar todos los beneficios que solo les dan a las instituciones de firmas o nombre maduros, porque es notorio que existe un apoyo deficiente para estos emprendimientos, lo cual deben de prestarle la atención necesaria para que ayuden a seguir aumentando la economía de los países en donde se instalen.

- No es suficiente tener los conocimientos respectivos para emprender un negocio también se necesita de ese espíritu de líder, ese don para soñar en grande y poder dirigir la empresa o negocio que se constituya cumpliendo todos los requisitos legales y permisos para su creación.

21 Vol. 5, núm. 2, abril 2019, pp. 03-27

21 Jorge Oswaldo Quevedo Vázquez, Lesi Vanessa Giler Escandón, Xavier Augusto Mantilla Crespo, Florencio Iván García Álvarez 
Cabe destacar que cada ser humano tiene su esencia y pensamiento propio, que se va formando a través del tiempo, cristalizando sus ideas para luego ponerlos en práctica, y así poder conocer el entorno en el que se maneja permitiéndole aprovechar todos los recursos necesarios para cumplir con sus metas y basarse en las ineficiencias, qué tal vez otros no las perciban, para garantizar una ganancia a corto o largo plazo, antes de emprender un negocio y así tener claro cuáles son las metas que se desean cumplir y los objetivos para los que se creó la empresa.

- Un líder o un emprendedor para tener éxito debe asumir como prioridad la innovación y la renovación permitiendo de esa manera ayudar a las empresas ya existentes para que puedan cumplir sus objetivos, así como tener la necesidad de tener visión para enfrentar los problemas futuros, producto de la necesidad que el mercado va a requerir, puesto que después de un tiempo viene otro amparando tal vez otro tipo de dificultades. Un empresario para garantizar un éxito total debe crear sus propios esquemas garantizando el uso de métodos modernos.

- Tanto la creación de empresas como la estrategia son disciplinas con ámbitos de trabajo propios. Queda claro, además, que son dos disciplinas complementarias, y que conjuntamente pueden generar trabajos teóricos y empíricos valiosos, como la capacidad para descubrir oportunidades, el conocimiento emprendedor y la habilidad para coordinar los recursos, tomados del campo de la creación de empresas.

- Las PYMES son programas públicos para resolver sus problemas de gestión interna, en la que los problemas relacionados con su gestión resalta la falta de capacitación y la de una cultura de innovación y avance tecnológico como los principales problemas asociados y, en principio, no son problemas la falta de liquidez, los costos de operación, la falta de competitividad, ni de calidad.

- Las PYMES se fortalecen como una posibilidad de mejoramiento de la competitividad, y por ello deben desarrollarse las condiciones internas de las empresas con el fin de generar un contexto político y económico, en el cual las mismas puedan perfeccionarse y superar las dificultades que su tamaño trae. 


\section{Referencias Bibliográficas}

Anokhin y Schulze. (2009). La corrupción política impide la innovación firme. Recuperado de: https://www.aeaweb.org/conference/2018/preliminary/powerpoint/zsHbR8Ki.

Araque (2009). Analisis de los factores que influyen en el emprendimiento y la sostenibilidad de las empresas del area urbana de la ciudad de Cuenca Ecuador. El perfil del emprendedor ecuatoriano. Recuperado de: https://www.researchgate.net/publication/313360043

Arteaga y Lasio, C. (2009). Empresas dinámicas en Ecuador factores de éxito y competencia de sus fundadores

Baird, I. (1988) Formalized planning in small business: Increasing strategic success Journal of Small Business Management, 31 (2): 38-51

Barceló y Pérez, (2003). Uso de las TICs en las PYMES en el noroeste de México. Universidad de Sonora-Chandler y Hanks.

Barney, J. (1991). Firm resources and sustained competitive advantage, Journal of Management, Vol17 (1), pp (99-120).

Bejarano, J. (1995). Las cadenas productivas y la competitividad. Cuadernos de Desarrollo Agrícola $1(1), 19-28$.

Belmar, C., y Maggi, C. (2010). Políticas e instituciones de fomento de las pyme en chile. En: Políticas de apoyo a las PYME en América Latina: entre avances innovadores y desafíos institucionales. Santiago: CEPAL, 2010. LC/G. 2421-P. p. 163-208.

Bettis, R y Hitt, M (1995). El Nuevo panorama competitivo. Recuperado de: https://onlinelibrary.wiley.com/doi/abs/10.1002/smj.4250160915

23 Vol. 5, núm. 2, abril 2019, pp. 03-27 
Birch, D. (1979). The Job Generation Process, mimeo, MIT. Program on Neighbourhood and Regional Change, February 1979.

Birley, S. (1987). Growth and Performance Contrasts between Types of Small Firms. Strategic Management Journal, vol. 11, núm. 7, pp. (535-557).

Brown, F., y Domínguez Villalobos, L. (2010). Políticas e instituciones de apoyo a la pequeña y mediana empresa en México. En: Políticas de apoyo a las PYME en América Latina: entre avances innovadores y desafíos institucionales. Santiago: CEPAL, 2010. LC/G. 2421-P. p. 299-342.

Chandler, Alfred D. y Takashi Hikino,(1994). "The large industrial enterprise and the dynamics of modern growth", en A. D. Chandler, F. Amatori y T. Hikino (editores), Big Business and the Wealth of Nations, Cambridge, Cambridge University Press, pp 24-62.

Díaz, A (2005) Impacto de un sistema ERP en una organización) Recuperado de: http://revistasinvestigacion.unmsm.edu.pe/index.php/sistem/article/download/3475/2862

García, González, y Murillo,(2017). Características de las PYMES

Gartner. (1989). Las Dimensiones Del Emprendimiento Empresarial - ResearchGate Recuperado de:

https://www.researchgate.net/publication/272306259_Las_Dimensiones_Del_Emprendimiento_Em presarial_La_Experiencia_De_Los_Programas_Cultura_E_Y_Fondo_Emprender_En_Medellln_Di mensions_of_the_Entrepreneurship_The_Experience_of_the_Programs_Cultura_E_and_

Gelderen, M., Bosma, N. y Thurik, R. (2001). Setting up a business in the Netherland: who starts, who gives up, who is still trying. Frontiers of Entrprneurship Research. Recuperado de http://www.babson.edu/entrep/fer/Babson2001/I/IG/IG.htm

Gelderen, M., Bosma, N. y Thurik, R. (2001). Setting up a business in the Netherland: who starts, who gives up, who is still trying. Frontiers of Entrprneurship Research. Recuperado de http://www.babson.edu/entrep/fer/Babson2001/I/IG/IG.htm 
Gómez, L y Hernández, (2009). Análisis del uso de nuevas tecnologías de información y comunicación en las PYMES de los sectores de calzado y marroquinería, joyeria, avícola y metalmecánico de Bucaramanga y su área metropolitana. (Tesis de grado). Bogotá, Colombia: Programa de Ingeniería en Informática, Pontifica Universidad Bolivariana. Recuperado 10/01/2014 de http://repository.upb.edu.co:8080/ jspui/bitstream/123456789/970/1/digital_19696.pdf

Greening, D y Johnson, R. (1996). Do managers and strategies matter a study in a crisis. Journal of management Studies. 33.1.pp (25-51).

Kachlami, H., y Yazdanfar, D. (2016). Determinants of sme growth: The influence of financing pattern. An empirical study based on swedish data. Management Research Review, 39(9), 966-986. doi: 10.1108/MRR-04-2015-0093

Kantis, H. (2004).Desarrollo y gestión de Pyme: aportes para un debate necesario. Universidad Nacional de General Sarmiento, Argentina.

Kent, C. (1990). Introduction: educating the heffalump. In Kent Eds. Entrepreneurship education: current developments, future directions. pp (1-26). New York, NY: Quorum Books

Kirzner, I. (1999). Competencia y función empresarial. Unión Editorial. Madrid. Reproducido el cap. II "El Empresario" en Lecturas de economía política vol. I. Unión Editorial, Madrid (1979). Traducción del original Competition and Entrepreneurship (1973), The University of Chicago Press.

Loutfi, M. (1991). Configuración y postulados básicos del trabajo por cuenta propia en Europa. Revista Internacional del Trabajo. Vol. 110(2), pp.(153-174).

Marshall, A. (1963). Principles of Economics: An Introductory, Press Oxford. U.K.

McClelland, D. (1961). The Achieving Society. New York: Free Press

25 Vol. 5, núm. 2, abril 2019, pp. 03-27 
O’Connor, R. (2013). Harmonizing Software Development Processes with Software Development Settings-A Systematic Approach. Systems, Software and Services Process Improvement. 364. Pp (167-178).

Pérez, M. (2004). La PyME en el Tolima: características y problemas. Cuadernos de investigación. Vol. 7. Universidad Coruniversitaria p. $(37-54)$

Peteraf (1993). Los pilares de la ventaja competitiva. Recuperado de: https://www.researchgate.net/publication/243771372_The_cornerstones_of_competitive_advantage _A_resource-based_view.

Porter, M. (1980). Competitive Strategy: Techinques for Analyzing Industries and Competitions. Austin, TX, Estados Unidos: Simón \& Schuster.

Quintana, C. (2001). Dimensiones del éxito de las empresas emprendedoras. Investigaciones Europeas de Dirección y Economía de la Empresa, 7(2), 139-158.

Solleiro, J. y Castañón, R (2005). Competitividad y sistemas de innovación, los retos para la inserción de México en el contexto global. Programa de Apoyo a Proyectos de Investigación e Innovación Tecnológica de la DGAPA-UNAM, México DF.

Sztulwark, S. (2010). Políticas e instituciones de apoyo a las pymes en la argentina. Políticas de apoyo a las pymes en América Latina, 45.

Thurik, P. (2001). Entrepreneurship and the business cycle. Review of Economics and Statistics. 94(4), pp. (1143-1156).

Valencia, M. (2007). La Competitividad un estudio en las PyMES desde la Gestión del Conocimiento. Ponencia presentada en la XLIV Asamblea anual 2009. Guayaquil, Ecuador. 2009, noviembre. 
Vásquez, R. (2007). Las MIPYMES y la Administración de Riesgos, en Rafael Regalado Hernández. Las MIPYMES en Latinoamérica. México: Red Latinoamericana de Investigadores en Administración.

Veciana, .M. (1999). Empresarilprocess de ceeació d'empreses, Revista Económica de Catalunya, 8. -- (1999). Creación de empresas como programa de investigación científica", Revista Europea de Direcci6n y Economía de la Empresa, 8(3): 11-36.

Wernerfelt, B. (1984). Inefficient Pre-Bargaining Search. Journal of Institutional and Theoretical Economics, Vol.168, pp (211-23).

York and Venkataraman, (2010). Estrategia, Etica y emprendimiento. Recuperado de: https://www.darden.virginia.edu/faculty-research/directory/s / venkataraman

Zamora, J. (2013). Las nuevas tecnologías y su impacto en la competitividad empresarial. Recuperado de: https://www.iese.edu/es/noticias/las-nuevas-tecnologias-y-su-impacto-en-la-competitividadempresarial/

Zuleta, J., et al.Alberto, L. (2011). Políticas e instituciones de apoyo a las pymes en Colombia. En: Apoyando a las pymes: políticas de fomento en América Latina y el Caribe. Santiago: CEPAL, 2011. p. 165-222. LC/R. 2180.

27 Vol. 5, núm. 2, abril 2019, pp. 03-27 